\title{
Non-T Non-B CALLA Negative Adult Acute Lymphoblastic Leukemia
}

National Cancer Institute

\section{Source}

National Cancer Institute. Non-T Non-B CALLA Negative Adult Acute Lymphoblastic

Leukemia. NCI Thesaurus. Code C114580.

Non-T non-B, CALLA negative acute lymphoblastic leukemia that occurs during adulthood. 